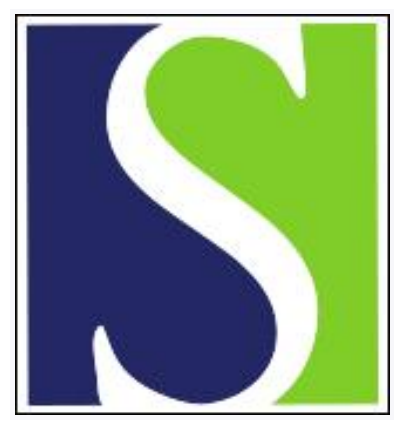

Scand J Work Environ Health 2002;28(3):176-183

https://doi.org/10.5271/sjweh.662

Issue date: Jun 2002

Individually fitted sports shoes for overuse injuries among newspaper carriers

by Torkki M, Malmivaara A, Reivonen N, Seitsalo S, Laippala P, Hoikka $\mathrm{V}$

Affiliation: Department of Orthopaedic Surgery, Helsinki University Central Hospital, Topeliuksenkatu 5, 00260 Helsinki, Finland. markus.torkki@netlife.fi

Key terms: footwear; individually fitted sports shoe; lower-limb overuse injury; newspaper carrier; overuse injury; randomized controlled trial; shock attenuation; shoe

This article in PubMed: www.ncbi.nlm.nih.gov/pubmed/12109557

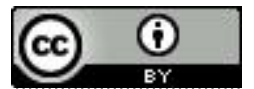




\title{
Individually fitted sports shoes for overuse injuries among newspaper carriers
}

\author{
by Markus Torkki, MD, ${ }^{1}$ Antti Malmivaara, PhD, ${ }^{2,4}$ Nina Reivonen, ${ }^{3}$ Seppo Seitsalo, PhD, ${ }^{4}$ Pekka Laippala, \\ $P h D,{ }^{5,6}$ Veijo Hoikka, $P h D^{7}$
}

Torkki M, Malmivaara A, Reivonen N, Seitsalo S, Laippala P, Hoikka V. Individually fitted sports shoes for overuse injuries among newspaper carriers. Scand J Work Environ Health 2002;28(3):176-183.

\begin{abstract}
Objectives The aim of the study was to determine the effectiveness of new, individually fitted sports shoes against overuse injuries to the lower limb among newspaper carriers.

Methods Patients $(\mathrm{N}=176)$ with lower-limb overuse injuries were randomly assigned to use new, individually adjusted footwear with good shock absorbing properties (test group = 86) or the subjects' own, used footwear (control group $=90$ ). The main outcome measurements were lower-limb pain intensity during walking, as rated on a visual analogue scale (0-100), number of painful days, subjective assessment of global improvement, foot fatigue, number of hyperkeratotic skin lesions and diagnosed overuse injuries, and costs of foot care as compared between the treatment groups.

Results At the 6-month follow-up there was a difference in favor of the test group with respect to lower-limb pain intensity and number of painful days, when compared with the control group. At 1 year, 53\% and 33\% of the test and control groups, respectively, thought they were better than at the time of the baseline examination (number needed to treat being 5 between the test and control groups). The test subjects had less foot fatigue and fewer hyperkeratotic skin lesions. There was no difference in the number of diagnosed overuse injuries between the groups. During the year of follow-up, the all-inclusive mean costs of foot care were USD 70 and USD 158 in the test and control groups, respectively.

Conclusions Individually adjusted shock-absorbing shoes offer slight health benefits for lower-limb overuse injuries. Proper shoes may decrease the need to use health care resources.
\end{abstract}

Key terms footwear, lower-limb overuse injuries, randomized controlled trial, shock attenuation, shoes.

Lower-limb overuse injuries are very common among people exposed to excessive walking or running during leisure-time activities or at work. It has been estimated that, in the Unted States, 10 million people jog or run frequently (1). Seventy percent of runners have chronic overuse injuries during their career (2). In a large epidemiologic study ( $\mathrm{N}=4358) 46 \%$ of the joggers had at least one overuse injury over a 1-year period, and $14 \%$ of them had sought medical advice for that injury (3).
In the shoe industry, the shock-absorbing properties of footwear have been studied intensively during the last few decades. Athletic footwear with good shock absorbing properties has been developed, and it is advertised as safeguarding against lower-extremity disorders (4). Nowadays athletic shoes constitute $34 \%$ of all shoes worn in the United States (5). In 1996, Americans spent USD 12 billion on about 340 million pairs of athletic shoes (5). However, there is negligible evidence that

Department of Orthopaedic Surgery, Helsinki University Hospital, Helsinki, Finland.

Department of Occupational Medicine, Finnish Institute of Occupational Health, Helsinki, Finland.

Department of Rehabilitation / Podiatry, Health Care and Social Services, Helsinki Polytechnic, Helsinki, Finland.

ORTON Orthopaedic Hospital, Invalid Foundation, Helsinki, Finland.

Helsinki, Finland; School of Public Health, University of Tampere, Tampere, Finland.

Research Unit, Tampere University Hospital, Tampere, Finland.

Department of Foot Surgery of Mehiläinen Hospital, Helsinki, Finland.

Reprint requests to: Dr Markus Torkki, Department of Orthopaedic Surgery, Helsinki University Central Hospital, Topeliuksenkatu 5, 00260 Helsinki, Finland. [E-mail: markus.torkki@netlife.fi] 
footwear with good shock absorbing properties really protects against injury (4). It has even been insinuated that current advertising is deceptive (4).

Biomechanical researchers have not yet arrived at a consensus on the best means with which to test for the biological consequences of various cushioning systems of footwear (6). In biomechanical studies it has been observed that the human locomotion system regulates shock absorption. When shoes with different shock-absorbing properties have been compared, no relation between shoe hardness and shock to the lower limb has been observed, as hardness of the shoe is associated with increased shock absorption in the body's own viscoelastic system (7). If the body's shock-absorption is minimized, the use of modern athletic footwear may even render the lower extremities more susceptible to injury (8).

To our knowledge, only one randomized trial on the effectiveness of shock-absorbing shoes for overuse injuries has been published (9). The work was performed among 390 military recruits, and it compared sports shoes with military boots.

Newspaper carriers in Finland comprise a group of employees who are highly exposed to walking and who have a high prevalence of lower-extremity overuse injuries. Using a randomized controlled trial, we thus set out to evaluate the effectiveness of new, individually fitted sports shoes among newspaper carriers executing their job mainly on foot, either walking or running.

\section{Subjects and methods}

\section{Selection and evaluation of the subjects}

The study was conducted using a population of Finnish newspaper carriers working for Leijonajakelu Ltd, which is a part of Helsinki Media Ltd, the biggest media company in Finland. For the study, we selected carriers who had suffered overuse injuries in their lower extremities and who did their work mainly on foot. The carriers' task included much climbing up and down stairs in apartment buildings because the papers are delivered individually to each household. To select the subjects, we first sent a questionnaire to all workers of Leijonajakelu ( $\mathrm{N}=2000)$ to determine whether the workers had had symptoms in the knee or distally during the last 3 months (table 1). Those who replied positively $(\mathrm{N}=260)$ were called for a clinical examination between April and May 1998 (figure 1). The inclusion criteria for the study were overuse injuries in the lower limbs: heel pain; plantar fascitis; metatarsalgia; inflammation of the metatarsophalangeal joints; achilles tendinopathy; tibialis peroneus, anterior or posterior tendinitis (or peritendinitis); anterior knee pain; and shin splint (table 2). The diagnosis was based on a clinical baseline examination, which was made by an orthopedic surgeon (MT). The work shoes were evaluated systematically by a foot therapist (NR), who also recorded all hyperkeratotic lesions on the plantar aspect of the foot. Only carriers who walked at least 3 kilometers or climbed at least 100 floors in their daily delivery route were included.

The carriers with new work shoes (shoes that had been used for 3 months or less) were excluded. Other exclusion criteria were (i) radicular pain (sciatic syndrome), (ii) menisceal rupture of the knee, and (iii) rheumatoid diseases. Subjects fulfilling the inclusion criteria were given written and oral information on the aims and content of the study in accordance with the Helsinki Declaration (10) before their decision as to whether to participate was requested. Thereafter, the patients gave their written consent, underwent a physical

Table 1. Frequency of diagnosed overuse injuries the 3 months preceding the baseline examination and the 12-month follow-up. ${ }^{a}$

\begin{tabular}{|c|c|c|c|c|c|c|c|c|}
\hline \multirow[b]{3}{*}{ Diagnosis } & \multicolumn{4}{|c|}{ Baseline examination } & \multicolumn{4}{|c|}{ 12-month follow-up } \\
\hline & \multicolumn{2}{|c|}{$\begin{array}{l}\text { Test group } \\
(\mathrm{N}=86)\end{array}$} & \multicolumn{2}{|c|}{$\begin{array}{l}\text { Control group } \\
(\mathrm{N}=90)\end{array}$} & \multicolumn{2}{|c|}{$\begin{array}{l}\text { Test group } \\
(\mathrm{N}=84)\end{array}$} & \multicolumn{2}{|c|}{$\begin{array}{l}\text { Control group } \\
(\mathrm{N}=90)\end{array}$} \\
\hline & $\mathrm{N}$ & $\%$ & $\mathrm{~N}$ & $\%$ & $\mathrm{~N}$ & $\%$ & $\mathrm{~N}$ & $\%$ \\
\hline \multicolumn{9}{|l|}{ Inflammation of } \\
\hline metatarsophalangeal joint & 22 & 26 & 25 & 28 & 8 & 10 & 15 & 17 \\
\hline Metatarsalgia & 27 & 31 & 23 & 26 & 3 & 4 & 9 & 10 \\
\hline Plantar fascitis & 25 & 29 & 22 & 24 & 8 & 10 & 5 & 6 \\
\hline Akilles tendinopathy & 33 & 38 & 35 & 39 & 13 & 16 & 20 & 22 \\
\hline Tibialis posterior tendinitis & 16 & 19 & 16 & 18 & 7 & 8 & 8 & 9 \\
\hline Tibialis anterior tendinitis & 4 & 5 & 7 & 8 & 3 & 4 & 0 & 0 \\
\hline Peroneus tendinitis & 21 & 24 & 21 & 23 & 7 & 8 & 16 & 18 \\
\hline Shin splint & 10 & 12 & 20 & 22 & 5 & 6 & 8 & 9 \\
\hline Anterior knee pain & 22 & 26 & 30 & 33 & 11 & 13 & 17 & 19 \\
\hline \multicolumn{9}{|l|}{ Subjects having at least } \\
\hline one overuse injury & 86 & 100 & 90 & 100 & 34 & 40 & 50 & 56 \\
\hline
\end{tabular}

a Some patients had more than one diagnosis. 


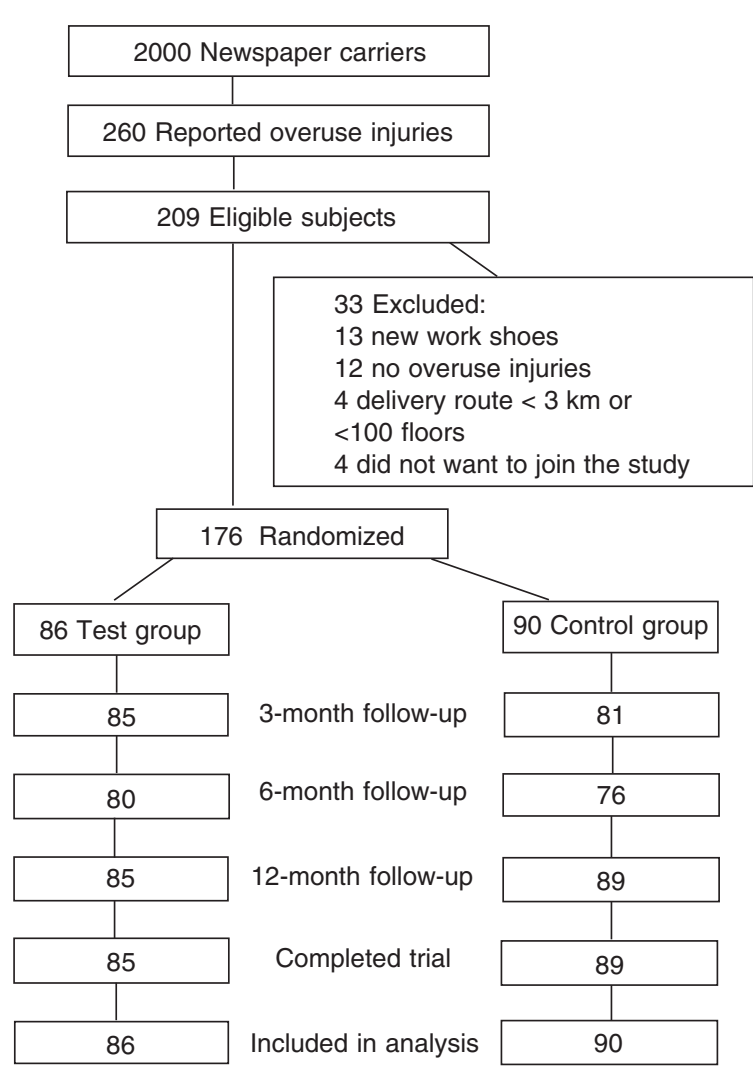

Figure 1. Patient flow chart. examination, and completed the questionnaires. The ethics committee of the Finnish Institute of Occupational Health approved the study protocol.

Before the randomization, the patients completed a baseline questionnaire, which was given to the research team immediately after the patients had answered it. The researchers analyzing the baseline and outcome data were blinded to the treatment protocols. Baseline data were gathered on potential confounders, effect-modifying factors, and factors related to lower-limb disorder (table 2). Duration of sick leave due to a lower-limb disorder was determined from the data given in the questionnaire.

\section{Randomization and intervention}

The randomization process was based on a list of numbers in a random number table. The block size for randomization, not previously known to the investigators, was 15 . To ensure an equal distribution of patients into the two groups, the patients were stratified according to age ( $<40$ and $>40$ years of age) and gender. After the patients who met the inclusion criteria had given their informed consent, one of the authors (NR) opened an envelope and gave each patient the protocol instructions. Patients randomized to the test group were fitted with new, fitting sports shoes with good shock attenuation

Table 2. Demographic and clinical characteristics of the study subjects at the time baseline examination.

\begin{tabular}{|c|c|c|c|c|c|c|}
\hline \multirow[b]{2}{*}{ Characteristic } & \multicolumn{3}{|c|}{ Test group $(\mathrm{N}=86)$} & \multicolumn{3}{|c|}{ Control group $(\mathrm{N}=90)$} \\
\hline & $\%$ & Mean & SD & $\%$ & Mean & SD \\
\hline \multicolumn{7}{|l|}{ Demographic features } \\
\hline Age (years) & . & 44 & 9.2 & . & 44 & 9.3 \\
\hline Female gender $(\%)$ & 42 & . & . & 44 & . & . \\
\hline Education high school or higher (\%) & 28 & $\cdot$ & . & 30 & . & $\cdot$ \\
\hline Body mass index $\left(\mathrm{kg} / \mathrm{m}^{2}\right)$ & . & 26 & 4.5 & . & 26 & 4.2 \\
\hline \multicolumn{7}{|l|}{ Work-related features } \\
\hline Daily walking distance at work (km) & . & 2.9 & 2.2 & . & 3.3 & 3.9 \\
\hline Number of floors in the apartment building on the delivery route & . & 145 & 97 & . & 142 & 92 \\
\hline \multicolumn{7}{|l|}{ Pain and disability } \\
\hline Pain intensity in lower limb a & . & 33 & 23 & . & 35 & 24 \\
\hline Duration of pain in the lower limbs (days during the last 3 months) & . & 35 & 32 & . & 40 & 34 \\
\hline Number of skin disorders in the plantar aspect of the feet & . & 5.7 & 3.7 & . & 5.8 & 3.6 \\
\hline Foot fatigue (days during the last week) & $\cdot$ & 2.0 & 1.0 & $\cdot$ & 2.1 & 1.1 \\
\hline Overuse injuries of the lower limb (\% of subjects) & 100 & 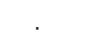 & . & 100 & . & . \\
\hline Number of diagnosed overuse injuries & $\cdot$ & 2.8 & 1.8 & $\cdot$ & 3.0 & 1.9 \\
\hline \multicolumn{7}{|l|}{ Sick leave (days during the last 3 months) } \\
\hline Due to foot problems & . & 1.1 & 2.9 & . & 3.1 & 10.5 \\
\hline Due to falls at work & . & 1.1 & 3.3 & . & 2.2 & 8.6 \\
\hline \multicolumn{7}{|l|}{ Work shoes } \\
\hline Price (USD) & . & 52 & 26 & . & 52 & 26 \\
\hline Age (months) & . & 11 & 11 & . & 10 & 8 \\
\hline Satisfaction with work shoes ${ }^{b}$ & . & 64 & 24 & . & 62 & 23 \\
\hline Ability to work ${ }^{c}$ & . & 83 & 13 & . & 82 & 15 \\
\hline
\end{tabular}

a Recorded on a 100-mm visual analogue scale scale from 0 (no pain at all) to 100 (unbearable pain).

b Recorded on a 100-mm visual analogue scale scale from 0 (totally unsatisfied) to 100 (totally satisfied).

${ }^{c}$ Recorded on a 100-mm visual analogue scale scale from 0 (total inability to work) to 100 (maximal work ability). 
properties. The patient's foot type was evaluated by a foot therapist using an electronic meter developed and recommended by the manufacturer, Karhu Sporting Goods Ltd, of the test shoes. There was a choice of shoes with a narrow or wide width, and with medial support (pronation type) or lateral support (supination type). The shore hardness value (given by the manufacturer) of the test shoes was 45 (SD 3) for all the test shoes, with the exception of the medial side of the pronation type of shoes (shore hardness value 55 (SD 3). The testing procedure took about 20 minutes. The test shoes were sent to the subjects in the test group within 6 weeks. They were instructed to wear the test shoes at work during the next 12 months and were informed that they could obtain a new pair of shoes if needed during the study. The patients in the control group were instructed to wear an analyzed pair of their own work shoes during the next 12 months. They were informed that they would be given a new pair of work shoes at the end of the study. All the patients were advised to contact one of the authors (NR) in the case of any problems concerning shoes during the study.

\section{Adherence and other interventions}

In the follow-up questionnaires, all the patients were asked how many floors they have in their work area, how many kilometers they walk per day at work and outside work, and whether they had received physician's, physiotherapist's, or podiatrist's health care services concerning lower limbs. They were asked if they had used the test shoes, their own analyzed work shoes, or some other shoes at work, approximately how many kilometers per day they had used these shoes outside work, and what shoes they had used on each day of the week prior to the questioning.

\section{Follow-up and outcome assessment}

The follow-up questionnaires were sent to the subjects 3 and 6 months after the randomization. The measured outcomes were lower-limb pain intensity, duration of pain, ability to work, foot fatigue, costs of foot care, and satisfaction with work shoes. Fall accidents at work and sick leave related to lower-limb disorders were also recorded.

At the 1-year follow-up, the patients were examined again and asked to complete another questionnaire. The measured outcomes were lower-limb pain intensity, duration of pain, ability to work, foot fatigue, costs of foot care, global assessment of improvement, satisfaction with work shoes, fall accidents at work, and sick leave in relation to lower-limb disorders. The number of hyperkeratotic skin lesions on the feet was recorded by one of the authors (NR) and overuse injuries by another
(MT). Of the authors, the former was not blinded to the study protocol, but the latter remained blinded throughout the study period.

\section{Economic analysis}

At the baseline examination the price of the analyzed shoes was requested in the questionnaire. Otherwise, the economic analysis was based on the responses to the 3month, 6-month, and 1-year follow-up questionnaires, which asked about the use of health care services for foot care. This information included visits to a physician and to a physiotherapist or foot therapist. The costs were calculated from the unit costs of these services in the Uusimaa Health District Area. The use of foot splints, braces, or orthoses was also recorded on the basis of the patients' own expenditure for them. Dollar costs were calculated at the 1998 exchange rate (USD 1 = FIM 5.096).

\section{Study population}

Altogether 176 subjects (aged 16-65 years) fulfilling the eligibility criteria were randomly assigned to the two treatment groups (figure 1). Eighty-six patients were randomized to the test group and 90 to the control group. The follow-up information was obtained 3 months later for 166 subjects (94\%); one subject was absent from the test group and 9 from the control group. After 6 months, information was obtained for 156 subjects $(89 \%)$; this time 6 subjects were missing from the test group and 14 from the control group. At the 1-year follow-up, information was obtained for 174 subjects (99\%); 1 subject was missing from both groups. The dropouts did not differ markedly from those remaining, as a whole, or among the two study groups. The frequency of diagnosed overuse injuries is shown in table 1. The demographic and clinical characteristics of the subjects are shown in table 2 . The two groups were similar with regard to all of the baseline characteristics.

\section{Adherence and co-interventions}

In the test group, $96 \%, 89 \%$, and $95 \%$ of the subjects reported that they had used the test shoes at the time of the 3-month, 6-month, and 1-year follow-ups, respectively. In the test group, the carriers who had used the test shoes reported in the 3-month, 6-month, and 1-year follow-ups that, in addition to work, they walked 2.4, 2.0 and 2.3 kilometers, respectively, per day with the shoes. Twenty-seven (31\%) of the 86 test group subjects got a new pair of shoes during the follow-up because the original pair wore out. In the control group, $60 \%$, $43 \%$, and $44 \%$ of the subjects at the 3-month, 6-month, and 1-year follow-ups, respectively, had used the same 
pair of shoes that had been analyzed by the study staff at the time of the baseline examination. Twelve, eight, and six percent of the carriers (at the 3-month, 6-month, and 1-year follow-ups, respectively) in the control group had used the test shoes (Karhu) during the study.

\section{Participant expectations}

To assess any possible preferences towards the treatments, we asked the participants, immediately after the randomization, whether they expected that their lower limbs would be better or not after the follow-up of 1-year. Sixty-seven percent of the test group and 18\% of the control group expected that their lower limbs would be better $(\mathrm{P}<0.001)$.

\section{Sample size and statistical analysis}

According to the power calculations, 68 subjects per treatment group were needed for the study in order to achieve a statistical power of 0.90 with an alpha of 0.05 (2-tailed). The calculations were made for pain during walking (the primary outcome) on an 0 to $100 \mathrm{~mm}$ visual analogue scale, with $15 \mathrm{~mm}$ as the clinically significant difference between the groups and on the assumption of a standard deviation of $15 \%$.

Efficacy variables were analyzed on an intention-totreat basis. The last observation was imputed for the patients who did not complete the study or who had missing values at 3,6 , or 12 months.

The data were primarily analyzed using an analysis of variance for repeated measures. For this setting, there is no clear choice for the error term to be used in the posthoc testing when the interaction is significant. Accordingly, our posthoc testing between the groups was based on $95 \%$ confidence intervals $(95 \% \mathrm{CI})$ constructed for the change over time.

Student's t-test was performed for the 3-month, 6month, and 1-year follow-up outcomes for the treatment

Table 3. Outcomes over the total study period.

\begin{tabular}{lrrc}
\hline & \multicolumn{3}{c}{ P-value } \\
\cline { 2 - 4 } Outcome & Group & Period & $\begin{array}{l}\text { Interaction } \\
\text { between } \\
\text { the group } \\
\text { and period }\end{array}$ \\
\hline Pain intensity in the lower limb & 0.077 & $<0.001$ & 0.311 \\
$\begin{array}{l}\text { Duration of pain in the lower limb } \\
\text { Ability to work }\end{array}$ & 0.071 & $<0.001$ & 0.835 \\
$\begin{array}{l}\text { Number of skin disorders in the } \\
\text { plantar aspect of the foot }\end{array}$ & 0.963 & 0.102 & 0.501 \\
$\begin{array}{l}\text { Foot fatigue (days during week prior } \\
\text { to questioning) }\end{array}$ & 0.217 & 0.520 & 0.045 \\
Satisfaction with work shoes & 0.039 & $<0.001$ & 0.014 \\
$\begin{array}{l}\text { Overall satisfaction } \\
\text { Slip resistance }\end{array}$ & 0.077 & 0.007 & 0.580 \\
Shock attenuation & 0.354 & $<0.001$ & 0.071 \\
Shoe comfort & $<0.001$ & 0.030 & 0.802 \\
\hline & 0.007 & 0.195 & 0.379 \\
\hline
\end{tabular}

groups (test group versus control). Cross-tabulations were analyzed using the chi-square test. Computations were carried out using NCSS 2000 (Jerry Hintze; Kaysville, Utah, United States) and Statistica/Win (Version 98; StatSoft; Tulsa, Okla) software programs.

\section{Results}

\section{Outcomes over the total study period}

The results of the analysis of variance for repeated measurements were summarized in terms of P-values in table 3. The reported results are based on the crude values. A data analysis was also carried out using transformations that also adjusted for the baseline values, but the results were identical. The significant interaction between the group and the period, indicating the significant treatment effect favoring the test group, was found for foot fatigue and for the number of hyperkeratotic skin lesions. The assessment of the slip resistance properties of the footwear also showed a trend towards significance.

\section{Three-month outcomes}

In the 3-month follow-up (tables 4 and 5), no a statistically significant difference was found between the groups for lower-limb pain intensity, duration of lowerlimb pain, ability to work, or foot fatigue. The subjects in the test group were more satisfied than the controls with the shock-absorbing properties, slip resistance, and comfort of their work shoes.

\section{Six-month outcomes}

In the 6-month follow-up (tables 4 and 5), lower-limb pain intensity, the number of the painful days, and foot fatigue favored the test group. The test group subjects gave a higher ranking to the shock-absorbing properties of their work shoes than did the controls.

\section{Twelve-month outcomes}

In the 12-month follow-up (tables 4 and 5), no statistically significant difference between the groups was found for lower-limb pain intensity, number of painful days, or ability to work. The global assessment by participant was better in the test group than in the control group (the number needed to treat being 5.0 between the test and control groups, $\mathrm{P}=0.025)$. The control group gave a higher rating for foot fatigue. The subjects in the test group had fewer hyperkeratotic skin lesions on the plantar aspect of the foot. No difference was found in the number of overuse injuries. The test group patients ranked the shock-absorbing properties and comfort 
Table 4. Outcomes in the test and reference groups at the time of the 3-, 6- and 12-month follow-ups.

\begin{tabular}{|c|c|c|c|c|c|c|c|c|c|c|c|c|c|c|}
\hline \multirow[b]{3}{*}{ Outcome } & \multicolumn{4}{|c|}{ 3-month follow-up } & \multicolumn{4}{|c|}{ 6-month follow-up } & \multicolumn{6}{|c|}{ 12-month follow-up } \\
\hline & \multicolumn{2}{|c|}{$\begin{array}{l}\text { Test group } \\
(\mathrm{N}=86)\end{array}$} & \multicolumn{2}{|c|}{$\begin{array}{l}\text { Control group } \\
(\mathrm{N}=90)\end{array}$} & \multicolumn{2}{|c|}{$\begin{array}{l}\text { Test group } \\
(\mathrm{N}=86)\end{array}$} & \multicolumn{2}{|c|}{$\begin{array}{l}\text { Control group } \\
(\mathrm{N}=90)\end{array}$} & \multicolumn{3}{|c|}{$\begin{array}{l}\text { Test group } \\
(\mathrm{N}=86)\end{array}$} & \multicolumn{3}{|c|}{$\begin{array}{l}\text { Control group } \\
(\mathrm{N}=90)\end{array}$} \\
\hline & Mean & SD & Mean & SD & Mean & SD & Mean & SD & $\%$ & Mean & SD & $\%$ & Mean & SD \\
\hline \multicolumn{15}{|l|}{ Pain and disability } \\
\hline Pain intensity in the lower limb & 29 & 27 & 32 & 26 & 27 & 22 & 36 & 28 & . & 23 & 25 & . & 29 & 25 \\
\hline (days during the follow-up period) & 23 & 27 & 27 & 30 & 21 & 26 & 30 & 34 & . & 20 & 27 & . & 26 & 32 \\
\hline $\begin{array}{l}\text { Ability to work }{ }^{b} \\
\text { Foot fatique (days during the }\end{array}$ & 79 & 23 & 81 & 18 & 80 & 20 & 81 & 19 & . & 83 & 17 & . & 82 & 21 \\
\hline & 1.3 & 2.1 & 1.6 & 2.3 & 1.0 & 1.9 & 1.8 & 2.3 & $\cdot$ & 0.76 & 1.7 & $\cdot$ & 1.6 & 2.4 \\
\hline plantar aspect of the foot & . & . & . & . & . & . & . & . & . & 5.4 & 3.2 & . & 6.5 & 3.4 \\
\hline Number of diagnosed overuse injuries & $\cdot$ & $\cdot$ & . & . & $\cdot$ & . & . & . & . & 1.4 & 1.5 & . & 1.1 & 1.3 \\
\hline \multicolumn{15}{|l|}{ Global assessment by patient $(\%)^{c}$} \\
\hline $\begin{array}{l}\text { Foot is better than } 1 \text { year ago } \\
\text { Foot is as good as } 1 \text { year ago } \\
\text { Foot is worse than } 1 \text { year ago }\end{array}$ & $\dot{.}$ & $\dot{.}$ & $\dot{.}$ & $\dot{.}$ & . & . & $\dot{.}$ & $\dot{.}$ & $\begin{array}{l}52 \\
32 \\
16\end{array}$ & $\dot{.}$ & $\dot{.}$ & $\begin{array}{l}33 \\
38 \\
29\end{array}$ & & $\dot{.}$ \\
\hline \multicolumn{15}{|l|}{ Satisfaction with work shoes ${ }^{d}$} \\
\hline Overall satisfaction & 73 & 23 & 67 & 24 & 67 & 25 & 63 & 24 & . & 71 & 26 & . & 64 & 26 \\
\hline Slip resistance & 71 & 24 & 63 & 23 & 55 & 29 & 55 & 24 & . & 60 & 28 & . & 58 & 27 \\
\hline Shock attenuation & 74 & 23 & 60 & 26 & 70 & 23 & 57 & 26 & . & 71 & 25 & . & 59 & 27 \\
\hline Shoe comfort & 79 & 20 & 69 & 22 & 75 & 22 & 70 & 21 & . & 76 & 23 & . & 67 & 25 \\
\hline Fall accidents at work ${ }^{\mathrm{e}}$ & $\cdot$ & $\cdot$ & $\cdot$ & $\cdot$ & $\cdot$ & $\cdot$ & $\cdot$ & $\cdot$ & . & 1.9 & 3.1 & . & 2.0 & 2.9 \\
\hline Foot-related sick leave ${ }^{e}$ & . & . & . & . & . & . & . & . & . & 3.9 & 11 & . & 9.4 & 24 \\
\hline
\end{tabular}

a Recorded on a 100-mm visual analogue scale from 0 (no pain at all) to 100 (unbearable pain).

${ }^{\mathrm{b}}$ Recorded on a 100-mm visual analogue scale from 0 (total inability to work) to 100 (maximal work ability).

c $\mathrm{P}=0.025$

${ }^{d}$ Recorded on a $100-\mathrm{mm}$ visual analogue scale from 0 (totally unsatisfied) to 100 (totally satisfied).

${ }^{\mathrm{e}}$ All subjects with any missing data were excluded, and thus the groups were smaller ( $\mathrm{N}=76$ in both groups) than in the other outcome analyses.

Table 5. Differences in the group means with their $95 \%$ confidence intervals $(95 \% \mathrm{Cl})$ for the outcomes of the test and reference groups at the time of the 3-, 6-, and 12-month follow-ups.

\begin{tabular}{|c|c|c|c|c|c|c|}
\hline \multirow[b]{3}{*}{ Outcome } & \multicolumn{6}{|c|}{ Test minus control group } \\
\hline & \multicolumn{2}{|c|}{ 3-month follow-up } & \multicolumn{2}{|c|}{ 6-month follow-up } & \multicolumn{2}{|c|}{ 12-month follow-up } \\
\hline & Mean & $95 \% \mathrm{Cl}$ & Mean & $95 \% \mathrm{Cl}$ & Mean & $95 \% \mathrm{Cl}$ \\
\hline \multicolumn{7}{|l|}{ Pain and disability } \\
\hline Pain intensity in the lower limb a & -2.5 & $-10-5.3$ & -9.1 & $-17--1.7$ & -5.8 & $-13-1.7$ \\
\hline $\begin{array}{l}\text { Duration of pain in the lower limb } \\
\text { (days during the follow-up period) } \\
\text { Ability to work } \\
\text { Foot fatigue (days during the last week) }\end{array}$ & $\begin{array}{l}-5.0 \\
-2.3 \\
-0.30\end{array}$ & $\begin{array}{l}-14-3.6 \\
-8.4-3.9 \\
-0.95-0.35\end{array}$ & $\begin{array}{l}-9.0 \\
-0.40 \\
-0.75\end{array}$ & $\begin{array}{c}-18--0.1 \\
-6.2-5.4 \\
-1.4--0.12\end{array}$ & $\begin{array}{l}-5.9 \\
0.68 \\
-0.84\end{array}$ & $\begin{array}{c}-15-2.9 \\
-5.1-6.4 \\
-1.5--0.22\end{array}$ \\
\hline \multicolumn{7}{|l|}{ Satisfaction with work shoes ${ }^{c}$} \\
\hline $\begin{array}{l}\text { Overall satisfaction } \\
\text { Slip resistance } \\
\text { Shock attenuation } \\
\text { Shoe comfort }\end{array}$ & $\begin{array}{c}6.5 \\
8.1 \\
14 \\
9.6\end{array}$ & $\begin{array}{r}-4.5-13 \\
1.1-15 \\
6.4-21 \\
3.4-16\end{array}$ & $\begin{array}{l}4.0 \\
-0.49 \\
13 \\
5.8\end{array}$ & $\begin{array}{l}-3.4-11 \\
-8.3-7.4 \\
6.0-21 \\
-0.62-12\end{array}$ & $\begin{array}{c}7.0 \\
1.3 \\
12 \\
8.3\end{array}$ & $\begin{array}{l}-0.75-15 \\
-6.9-9.6 \\
4.0-20 \\
1.2-15\end{array}$ \\
\hline Number of skin disorders in the plantar aspect of the foot & $\cdot$ & $\cdot$ & $\cdot$ & $\cdot$ & -1.1 & $-2.1--0.12$ \\
\hline Number of diagnosed overuse injuries & . & . & . & . & 0.27 & $-0.13-0.67$ \\
\hline Fall accidents at work ${ }^{d}$ & . & . & . & . & -0.039 & $-1.0-0.94$ \\
\hline Foot-related sick leave ${ }^{d}$ & . & . & . & . & -5.5 & $-11-0.47$ \\
\hline
\end{tabular}

a Recorded on a 100-mm visual analogue scale scale from 0 (no pain at all) to 100 (unbearable pain).

${ }^{b}$ Recorded on a 100-mm visual analogue scale from 0 (total inability to work) to 100 (maximal work ability).

${ }^{c}$ Recorded on a $100-\mathrm{mm}$ visual analogue scale from 0 (totally unsatisfied) to 100 (totally satisfied).

${ }^{d}$ All subjects with any missing data were excluded, and thus the groups were smaller ( $\mathrm{N}=76$ in both groups) than in the other outcome analyses. 
of the work shoes as better than those of the control group. The number of fall accidents at work was similar in the two groups. There was a trend towards less sick leave due to lower-limb injuries in the test group over the total study period (tables 4 and 5). There was no difference between the two groups in leisure-time walking activity [mean daily walking distances 2.3 (SD 2.8) and 2.3 (SD 2.7) kilometers in the test and control groups, respectively].

\section{Costs and use of services}

During the 1-year follow-up period the controls spent an average of USD 158 for medical foot care, whereas the corresponding sum was USD 70 in the test group (table 6). There was no statistically significant difference between the two groups in the amount of the sick leave because of lower-limb injuries over the total study period.

\section{Discussion}

All the newspaper carriers recruited for the study had some kind of overuse injury of the lower limb at the beginning of the study. They carried out their job mainly on foot. During each day the carriers climbed an average of 144 flights of steps in apartment buildings and walked 3.1 kilometers. In addition they walked every day an average of 2.3 kilometers with the test shoes. A population with lower-limb injuries and exposure to excessive stress on the lower limbs was chosen in order to be able to show the maximal health benefit of the individually fitted shock-absorbing shoes.

However, despite the strengths in the study design, we encountered some insurmountable limitations inherent in this particular study object. For ethical reasons

Table 6. Mean costs of foot care over a 12-month period. ${ }^{\text {a }}$

\begin{tabular}{|c|c|c|}
\hline Type of cost & $\begin{array}{l}\text { Test group } \\
(\mathrm{N}=78)\end{array}$ & $\begin{array}{l}\text { Control group } \\
\qquad(\mathrm{N}=74)\end{array}$ \\
\hline $\begin{array}{l}\text { Study treatments } \\
\text { Health services for foot care }\end{array}$ & 88 & $52^{b}$ \\
\hline Visits to physician ${ }^{c}$ & 66 & 119 \\
\hline $\begin{array}{l}\text { Visits to a physiotherapist or } \\
\text { foot therapist }{ }^{d}\end{array}$ & 4.5 & 39 \\
\hline Total costs & 159 & 210 \\
\hline \multicolumn{3}{|c|}{$\begin{array}{l}\text { a Costs are in United States dollars in 1998. The sums are expressed as } \\
\text { the mean price per patient. All the subjects with any missing data were } \\
\text { excluded, and thus the groups were smaller than in the other outcome } \\
\text { analyses. } \\
\text { b Price at the time of purchase. } \\
\text { c These costs include visits to physicians during the follow-up period. } \\
\text { d These costs include visits to physiotherapists or foot therapists } \\
\text { because of foot problems during the follow-up period. }\end{array}$} \\
\hline
\end{tabular}

we could not offer "standard" shoes with poor shockabsorbing properties for the control group, as they could be considered unhealthy for persons suffering from lower-extremity overuse injuries. Therefore, we designed our study to answer the practical question of whether it is of benefit to offer new, individually fitted shock-absorbing shoes to mobile persons having lower-limb problems who currently use old, worn shoes, as was the case among our subjects.

Maintaining a clear contrast between the two groups was another problem for the same reasons. In the test group, the subjects got a new pair of shoes if the old pair was totally worn out, but the persons in the control group could not be given the same offer. Our study indicates that the wear and tear on shoes is very high among newspaper carriers; even in the test group with new shoes at the beginning of the study, $31 \%$ of the test subjects needed a new pair during the 1-year follow-up, because the old pair was worn out. In the control group, $44 \%$ of the subjects still used the same shoes at the 1-year follow-up as at the baseline, but more than half had bought new shoes. Therefore, the intervention contrast was not completely maintained.

The randomization resulted in a good comparability in the baseline characteristics between the two groups. The loss to 1 -year follow-up was only $1 \%$, and those from whom no follow-up data were available did not differ in terms of baseline characteristics from those who attended the follow-up. As double blinding was not feasible, we assessed the expectations for recovery after randomization, as well as satisfaction with the shoes. The subjects' expectations for the interventions at the baseline were more favorable in the test group, which might somewhat have influenced the subjective assessments of outcome towards overestimating the shoe effects.

In both groups, the number of diagnosed overuse injuries diminished during the follow-up. This finding, which may be due to a favorable natural course of these injuries, underlines the importance of controlled studies when studying the health effects of footwear. The author diagnosing the injuries was blinded to the study protocol, and no difference was found between the groups. The number of painful days, global assessment of improvement, foot fatigue, and the number of hyperkeratotic skin lesions on the feet favored the test group, but the observer recording the lesions was not blinded. The main outcome, lower-limb pain intensity, favored the test group only at the 6-month follow-up. However, at this phase, 14 subjects were missing from the control group compared with 6 missing patients in the test group. The last observation was imputed for the missing subjects in the intention-to-treat analysis. Because the symptoms in both groups tended to diminish during the study, the observed difference at the 6-month 
follow-up may have been due to the fact that the outcome values of the missing subjects were imputed from the less favorable values of the 3-month follow-up. Economic assessment showed that the cost of the test shoes exceeded that of the shoes in the control group, but during the follow-up the subjects in the control group spent more money on medical foot care. There was a trend towards less lower-limb-related sick leave in the test group.

In our study some outcomes favored the test group. However, this outcome cannot be explained only by good shock-absorbing properties of the test shoes. Despite randomization, there were several differences between the two groups during the study. The shoes of the test group were new and of proper size. In addition, the substantial differences in expectations should be considered when the results are evaluated; $67 \%$ of the test group expected their feet to improve compared with $18 \%$ of the control group.

The differences in the outcome between the groups are rather small. Despite the limitations of our study, it seems probable that new, individually fitted sports shoes with good shock-absorbing properties do not offer extraordinary health benefits for persons having lowerlimb overuse injuries. However, the decreased use of health care resources may indicate fewer complaints among the test group participants, and more frequent use of health care services may have brought health benefits for the control group.

In conclusion, based on the results of this trial, individually fitted shock-absorbing shoes seem to offer only rather small health benefits to subjects exposed to daily walking and having lower-limb overuse injuries. The effect is smaller than one would anticipate from the current unsubstantiated advertising. However, proper footwear may lead to some health benefits and decreased use of health care resources. Although the task of studying the efficacy of shoes within a validly designed study is a difficult one, there is an obvious need for more evidence in this field.

\section{Acknowledgments}

We are indebted to manager Seppo Kallio, Karhu Sporting Goods Ltd; managers Anne Nyysti and Timo Savolainen, Leijonajakelu Ltd, and secretary Monika Fre- driksson for their help. We would also like to thank Jeremy Dallyn for the English revision of the text.

This work was supported by a grant from the Professor Kallio Foundation and from the 100-year Foundation of Helsingin Sanomat

\section{References}

1. Nicholas JA. Clinical observations on sports-induced softtissue injuries in sports-induced inflammation. In: Leadbetter WB, Buckwalter JA, Gordon SL, editors. Sports-induced inflammation. Park Ridge (IL): American Academy of Orthopaedic Surgeons, 1989.

2. Sheehan GA. An overview of overuse syndromes in distance runners. In: Milvy P, editor. The marathon: physiological, medical, epidemiological and psychological studies. Ann NY Acad Sci 1977;301:877.

3. Marti B. Relationships between running injuries and running shoes. Results of a study of 5000 participants of a 16-km run — The May 1984 Berne "Grand Prix". In: Segesser B, Pferringer W, editors. The shoe in sport. Chicago (IL): Year Book Medical Publishers, 1989.

4. Robbins S, Waked E. Hazard of deceptive advertising of athletic footwear. Br J Sports Med 1989;31:299-303.

5. Winkelmann T. Does everybody need a running shoe? Stride 1997:4:14-8.

6. Clarke TE, Frederick EC, Cooper LB. Biomechanical measurement of running shoe cushioning properties. In: Nigg BM, Kerr BA, editors. Biomechanical aspects of sport shoes and playing surfaces. Calgary (Canada): Biomechanics Laboratory, 1983.

7. Nigg BM, Herzog W, Read LJ. Effect of viscoelastic shoe insole on vertical impact forces in heel-toe running. Am J Sports Med 1988;16:70-76.

8. Robbins SE, Gouw GJ. Athletic footwear and chronic overloading. Sports Med 1990;9:76-85.

9. Milgrom C, Finestone A, Shlamkovitch N, Wosk J, Laor A, Voloshin A, et al. Prevention of overuse injuries of the foot by improved shoe shock attenuation. Clin Orthop 1992;281: 189-92.

10. World Medical Association. World Medical Association declaration of Helsinki: ethical principles for medical research involving human subjects. JAMA 2000;23:3043-5.

Received for publication: 11 October 2001 